\title{
ESCALAMIENTO DE UN FOTOBIORREACTOR A NIVEL PILOTO PARA LA PRODUCCIÓN DE BIOMASA
}

\author{
PhOtobioreactor SCALE UP TO PILOT LEVEL FOR BIOMASS PRODUCTION
}

Diego Rubio Fernández*

Juan Sandoval Herrera**

Jenny P. Ruiz Díaz***

Jhonnathan C. Tovar Salamanca****

Recibido: 11 de enero de 2017

Aceptado: 25 de abril de 2017

\section{Resumen}

El escalamiento de fotobiorreactores implica que una relación intrínseca entre los parámetros que intervienen en el diseño de estos dispositivos y la fisiología del microorganismo determina una correcta operación del sistema. El modelo desarrollado en esta investigación permite cuantificar la influencia del parámetro luz en el crecimiento de la microalga Chlorella vulgaris a medida que varía el diámetro del reactor, en este caso, un fotobiorreactor tubular tipo airlift, con el fin de incrementar la productividad de biomasa microalgal partiendo de baja concentración inicial. El modelo permitió lograr un escalamiento de 7 a $50.2 \mathrm{~L}$, con un incremento menor en la productividad de biomasa del $100 \%$, aproximadamente.

Palabras clave: fotobiorreactor; luz; Chlorella vulgaris; sustratos; simulación

\begin{abstract}
Photobioreactor scaling implies that an intrinsic relationship between the design parameters and physiology of the organism determines an appropriate system operation. In this study, the model allows to evaluate the influence of light parameter into the growth of the microalgae Chlorella vulgaris as the diameter of the reactor increases, for an airlift FBR, with the aim of obtaining the highest biomass productivity from a low initial concentration. The model allowed a scaling from 7 to 50.2 liters with an increment of about $100 \%$ in its production rate.
\end{abstract}

Keywords: Chlorella vulgaris; light; photobioreactor, simulation, substratum

\footnotetext{
* Biólogo, magíster en Ciencias, Estudiante del Doctorado en Biotecnología (Universidad Nacional de Colombia). Docente investigador, grupo de investigación BIOTECFUA, Departamento de Investigaciones, Fundación Universidad de América.diego.rubio@investigadores.uamerica.edu.co

** Ingeniero químico, magíster en Formulación y Tecnología del Producto. Docente investigador, grupo de investigación BIOTECFUA, Programa de Ingeniería Química, Fundación Universidad de América.

juan.sandoval@profesores.uamerica.edu.co

${ }^{* * *}$ Estudiante coinvestigador, grupo de investigación BIOTECFUA, Programa de Ingeniería Química, Fundación Universidad de América Jenny.ruiz@estudiantes.uamerica.edu.co

${ }_{* * * *}$ Estudiante coinvestigador, grupo de investigación BIOTECFUA, Programa de Ingeniería Química, Fundación Universidad de América jhonnathan.tovar@estudiantes.uamerica.edu.co
} 


\section{INTRODUCCIÓN}

El escalamiento tiene diversas aplicaciones industriales o de investigación. Se pueden lograr grandes beneficios a partir de la simulación a pequeña escala de nuevas condiciones que se vayan a implementar a nivel industrial, como el cambio de valores en parámetros del proceso, el uso de nuevos equipos e, incluso, la viabilidad de una nueva sección de operación o de una planta de producción en su totalidad (Anaya-Durand y Pedroza-Flores, 2008).

Un propósito más amplio del escalamiento es la selección de las condiciones de diseño y de procedimientos operacionales para asegurar que el efecto de las diferentes variables del proceso sea el mismo en unidades de diferente tamaño. Por ejemplo, propiedades como la hidrodinámica y transferencias de masa se utilizan habitualmente como criterios para el escalamiento de biorreactores; igualmente, algunas de las principales variables se mantienen constantes, como la potencia de agitación por volumen de reactor, el coeficiente de transferencia de masa volumétrica, o el tiempo de mezclado (Najafpour, 2015).

Sin embargo, el escalado de fotobiorreactores requiere un mayor grado de complejidad, ya que debe tenerse en cuenta la heterogeneidad de la distribución de la intensidad de la luz dentro del reactor. Hay dos fenómenos físicos relacionados con la luz que interactúan y se contrarrestan mutuamente dentro del biorreactor: la absorción por parte de los pigmentos celulares y la dispersión generada por la totalidad de la biomasa. Factores como la geometría del reactor y el nivel de mezclado pueden generar zonas con niveles diferentes de iluminación, algunas, incluso, por debajo del punto de compensación para la fotosíntesis. Por lo tanto, un aspecto crucial del diseño de un fotobiorreactor es construir un modelo matemático que describa la interacción entre la disponibilidad de luz y el crecimiento celular (Vernerey, Albiol, Lasseur y Godia, 2001). Posten (2009) realiza una revisión completa del tema del diseño de fotobiorreactores e incluye la definición de los principales términos relacionados con la interacción de la luz y el crecimiento celular; igualmente, establece que la simulación es un método eficiente para entender la dinámica del sistema desde los cambios fisiológicos del cultivo y obtener resultados de producción al escalar sin necesidad de realizar el proceso físicamente. Esto genera que el proceso de modelamiento sea una herramienta que permita evaluar los bioprocesos a escalas industriales antes de llevar a cabo su puesta en marcha.

Esta investigación seleccionó un diseño base de fotobiorreactor, que efectivamente está funcionando en el grupo de investigaciones, para llevar a cabo su escalamiento por simulación matemática. En este proceso se escogió la variable productividad de biomasa como respuesta al fenómeno de la absorción de la luz; se definió un modelo de escalamiento, basado en las referencias bibliográficas más acordes con la especie a estudiar; se desarrolló el modelo para la especie en particular, y, finalmente, se llevó a cabo la simulación para encontrar el tamaño de reactor adecuado y lograr, partiendo del diseño base, la mayor productividad posible con la menor concentración de biomasa inicial.

\section{Métodos}

El proceso de escalamiento a nivel de bioprocesos implica considerar la relación existente entre las variables de diseño y los factores microbiológicos, de tal forma que las condiciones provistas para el cultivo se mantengan en los rangos adecuados para una producción y un rendimiento elevados. Para poder definir las relaciones entre variables de diseño y microbiológicas se estableció un proceso con diferentes fases.

\section{Definición del diseño base}

Considerando que el fotobiorreactor (FBR) es la unidad central de operación del proceso en base a microalgas, fue necesario escoger un diseño específico entre los múltiples FBR. Para el proceso 
presentado se consideró que la geometría de los fotobiorreactores tubulares era la adecuada para proponer el escalamiento. Es decir, el proceso de modelado considera la geometría cilíndrica que puede aplicarse a FBR tubulares, airlift o columnas de burbujeo.

\section{Selección de la variable a escalar o variable de escalamiento}

La variable de escalamiento se seleccionó a partir del fenómeno de la trasferencia lumínica en fotobiorreactores, que se ha considerado como la principal limitante de la productividad en procesos basados en microalgas. La luz es el factor más importante en el crecimiento de organismos fotosintéticos (Jeon, Cho y Yun, 2005), ya que sin este elemento sería imposible el proceso mismo y, por lo tanto, no habría crecimiento celular. Por tal razón, es necesario conocer bien la relación existente entre la variable luz y el crecimiento celular.

\section{Definición de un modelo de escalamiento}

Se seleccionó un modelo de escalamiento que considera como elemento principal la transferencia lumínica en el interior de los fotobiorreactores tubulares. Asimismo, se determinaron aspectos como la disponibilidad de información secundaria para parametrizar el modelo. El escalamiento de biorreactores se puede hacer de manera semiempírica, es decir, aumentando el volumen del reactor y evaluando los resultados obtenidos (Vanags, Kunga, Dubencovs, Galvanauskas y Grīgs, 2015). Este procedimiento puede mejorar algunas veces la productividad sin que sea necesario controlar parámetros como $\mathrm{pH}$, agitación o temperatura. Sin embargo, el método semiempírico es costoso y no muy efectivo. Otra alternativa es buscar un modelo que represente de la mejor manera posible los parámetros involucrados, para así verificar los resultados que se obtendrían al aumentar el volumen del cultivo sin que exista la necesidad de llevar el escalamiento del reactor a la práctica.

Un ejemplo de este tipo de escalado que parte de la luz es el de Vernerey et ál. (2001), el cual toma el modelo desarrollado por Cornet, Dussap y Dubertret (1992) (que se emplea para una geometría específica) para adecuarlo a la producción de biomasa de la microalga Chlorella vulgaris.

\section{DesarRollo y ANÁlisis deL MOdelo SELECCIONADO}

\section{Transferencia de luz en el FBR}

Cornett et ál. (1992) consideran un modelo de geometría monodimensional basado en leyes de transferencia de energía radiante en medios sólidos y, posteriormente, en medios líquidos. Las primeras teorías relacionadas con los fenómenos de absorción y dispersión de la luz en medios sólidos parten de un modelo monodimensional que se fundamenta en leyes de transferencia de energía radiante. Ejemplo de esto es el trabajo de Barron (1986), el cual se aplicó al campo de los pigmentos de los suelos. Por su parte, van Liere y Mur (1979) resumieron algunos estudios anteriores y publicaron sus resultados de la evaluación de la cinética de crecimiento de una microalga limitada por la disponibilidad de luz.

El fenómeno de transferencia de luz dentro de un biorreactor involucra dos factores: absorción por los pigmentos celulares y dispersión provocada por la biomasa. Del balance energético resultará la energía realmente disponible para el proceso fotosintético y el crecimiento celular.

Según Yun y Park (2003), existen cuatro modelos para cada tipo de parámetro lumínico de la fotosíntesis algal: los APFD (average photon flux density), que dependen de la densidad de flujo de fotones promedio; los LPFD (local photon flux density), en los que la actividad de cada célula miroalgal depende de la densidad de fotones que llega a esta; los APAR (average photon absortion rate), que usan la velocidad de absorción de fotones promediada en el volumen, y los LPAR (local 
photon absortion rate), que relacionan la actividad fotosintética de cada célula con la velocidad de absorción de fotones por parte de la célula.

Este trabajo parte de un modelo tipo LPFD (modelo que se ha aplicado a diferentes geometrías de fotobioreactores), en el que cada célula responde a la densidad de flujo de fotones que llega a su posición. Para poder obtener el modelo, llevar a cabo la simulación y, finalmente, realizar el escalamiento se parte de los dos fenómenos involucrados en la transferencia de la luz a las células: la absorción y la dispersión.

\section{Ecuaciones del modelo}

El modelo parte de las ecuaciones de Lambert-Beer y la hipótesis de Schuster para lograr simplificaciones en el balance de energía y desarrollar el modelo matemático propuesto. Estas simplificaciones sostienen que la luz se distribuye de manera isotrópica a través del reactor y que los fenómenos de absorción y dispersión, expresados por las constantes $E_{a}$ y $E$, respectivamente, son independientes entre sí. También considera que el flujo de fotones es monodimensional (Cornet, Dussap y Dubertret, 1992).

El flujo radiante, $F_{r}$, se compone de dos flujos opuestos: $F_{r}^{+}$y $F_{r}^{-}$. Cada partícula, es decir, cada célula, absorbe y dispersa parte de la radiación que llega a esta. El balance de energía disponible que llega a cada célula se puede expresar de la siguiente manera:

$$
\begin{aligned}
& \frac{d r F_{r}^{+}}{d r}=-E_{a} C_{x} r F_{r}^{+}+\frac{1}{2} E_{s} C x r\left(F_{r}^{-}-F_{r}^{+}\right) \\
& \frac{d r F_{r}^{-}}{d r}=E_{a} C_{x} r F_{r}^{-}+\frac{1}{2} E_{s} C_{x} r\left(F_{r}^{-}-F_{r}^{+}\right)
\end{aligned}
$$

Donde $E_{a}$ corresponde al coeficiente de absorción en masa; $E_{s}$, al coeficiente de dispersión en masa $\left(\mathrm{m}^{2} / \mathrm{kg}\right) ; r$, al distancia radial al centro del reactor, y $C_{x}$, a la concentración celular en un punto dado $\left(\mathrm{kg} / \mathrm{m}^{3}\right)$. El modelo es válido si se asume que el flujo radiante incidente en la pared externa del reactor, es decir cuando $r=R$ (R, es el radio total).

La intensidad media de luz, $J_{r}$, se relaciona con el flujo de energía radiante por medio de la siguiente ecuación:

$$
J_{r}=\frac{1}{4 \pi}\left(F_{r}^{+}-F_{r}^{-}\right)
$$

Ahora, la relación entre la energía radiante disponible, $4 \pi J_{r}$, y el flujo de energía radiante proporcionada al reactor desde su superficie externa, $F_{r}$, la establece Vernerey et ál. (2001) para un reactor de geometría cilíndrica:

$$
\frac{4 \pi J_{r}}{F_{r}}=\frac{1}{Z} \frac{2 \cosh (\boldsymbol{\delta} z)}{\cosh (\boldsymbol{\delta})+\alpha \sinh (\boldsymbol{\delta})}
$$

Donde $Z$ es un parámetro adimensional que relaciona una distancia radial, $r$, con el radio del reactor, $R$. Los parámetros $\alpha$ y $\delta$ se definen como funciones de los coeficientes de absorción, $E_{a}, y$ de dispersión de la luz, $E$, de acuerdo con las siguientes expresiones: 


$$
\begin{gathered}
\alpha=\sqrt{\frac{E_{a}}{E_{a}+E_{S}}} \\
\delta=\left(E_{a}+E_{S}\right) \alpha C_{x} R
\end{gathered}
$$

Ahora bien, la velocidad volumétrica de energía radiante absorbida, $A$, se calcula por medio de:

$$
\frac{\langle A\rangle}{\left(2 F_{R} / R\right)}=\frac{2 \alpha \sinh (\delta)}{\cosh (\delta)+\alpha \sinh (\delta)}
$$

Donde $F_{R}$ es la energía radiante que llega al reactor en $r=R$.

Igualmente, según las ecuaciones 3,4 y 7 , es necesario conocer el flujo radiante que llega a la pared del reactor. $Y$ dado que experimentalmente esto no es un procedimiento complejo, se debe usar una esfera fotométrica en el centro del reactor para determinar ese flujo radiante, $E_{b}$, en $\mathrm{W} / \mathrm{m}$. La energía radiante disponible en el centro del reactor se relaciona con el flujo de energía radiante en la pared por medio de:

$$
\frac{E_{b}}{F_{R}}=\frac{1}{Z_{b}} \frac{1}{\cosh (\delta)+\alpha \sinh (\delta)}\left[\pi I_{0}\left(\delta Z_{b}\right)\right]
$$

Donde $Z_{b}=r_{b} / R$ es la relación de radio de la esfera al radio del reactor, y $I_{0}$ es una función de Bessel modificada que equivale a:

$$
I_{0}\left(\delta Z_{b}\right)=\sum_{k=0}^{\infty} \frac{\left(\frac{\delta Z_{b}}{2}\right)^{2 k}}{k ! \Gamma(k+1)}
$$

Para bajos valores de $\delta$, bajas concentraciones de biomasa, la ecuación 8 se reduce a:

$$
\frac{E_{b}}{F_{R}}=\frac{1}{Z_{b}} \frac{1}{\cosh (\delta)+\alpha \sinh (\delta)}\left[1+\left(\frac{\delta Z_{b}}{2}\right) 2\right]
$$

Entonces, la $\mathrm{E}_{\mathrm{b}}$ se determina experimentalmente con la esfera fotométrica. Con la ecuación 10 se halla $F_{R}$ y luego este valor se puede reemplazar en las ecuaciones 4 y 7 a fin de encontrar el flujo de energía radiante disponible en un punto a una distancia $r$ del centro del reactor.

Aun si toda la energía que llega a la pared del reactor se distribuye homogéneamente dentro de este y en todo su volumen, se crean zonas oscuras dentro del reactor por la sola presencia de las células, cuyos pigmentos absorben esa energía. El crecimiento, que es el objetivo del modelo, se relaciona con la intensidad media de luz por la siguiente expresión general:

$$
\left\langle\mu^{\prime}\right\rangle=\frac{1}{V}\left(\int \mu_{M}^{\prime} C_{x} \frac{4 \pi J}{K_{J}+4 \pi J} d V\right) V
$$


A su vez, la solución para una geometría cilíndrica es, aproximadamente:

$$
\left\langle\mu^{\prime}\right\rangle \approx \frac{1}{\pi\left(R^{2}-R_{2}^{2}\right)} \int_{R_{2}}^{R} 2 \pi \mu_{M}^{\prime} \frac{4 \pi J_{r}}{K_{J}+4 \pi J_{r}} r d r
$$

En las ecuaciones 11 y 12, $\left\langle\mu^{\prime}\right\rangle$ es la tasa de crecimiento volumétrico $\left(\mathrm{kg} / \mathrm{m}^{3} . \mathrm{h}\right) ; \mathrm{K}_{\mathrm{j}}$ es la constante de saturación media $\left(\mathrm{W} / \mathrm{m}^{2}\right) ; \mu_{M}{ }^{\prime}$ es la máxima velocidad de crecimiento microalgal $\left(\mathrm{h}^{-1}\right) ; \mathrm{R}$ es el radio del reactor total, $\mathrm{y}_{2}$ es el radio de la fracción iluminada del reactor, es decir, la zona en la cual el nivel de iluminación es suficiente para permitir el proceso fotosintético. Cp es el nivel mínimo de energía en el cual la fotosíntesis es ineficiente. Al contrario de lo que ocurre en la zona oscura, en la fracción iluminada el crecimiento microalgal es exponencial.

En esta parte del proceso de cálculo, el objetivo es conocer la fracción iluminada para de este modo obtener el máximo crecimiento. Ya que el modelo se aplica a un fotobiorreactor tipo airlift que consta de dos cilindros concéntricos, la geometría que se aplica es cilíndrica. Por tanto, la fracción iluminada es:

$$
\gamma=\frac{R_{2}^{\prime 2}}{R^{2}}+\frac{R^{2}-R_{2}^{2}}{R^{2}}
$$

Teniendo en cuenta la fracción iluminada del reactor y el contenido de ficocianinas, $C_{f i}$ el crecimiento volumétrico promedio, $\langle R\rangle$, queda definido como:

$$
\langle R\rangle=\left\langle\mu^{\prime}\right\rangle \gamma C_{f_{c}}
$$

A manera de resumen del procedimiento de cálculo, el primer paso consiste en obtener los datos propios de la especie de microalga: $C_{p}, C_{f}, E_{a}$ y $E_{s}$. Se determina el $E_{b}$ por medio de la esfera fotométrica y con un valor de concentración de biomasa se fija $\gamma$. Posteriormente, se halla $\left\langle\mu^{\prime}\right\rangle$ y finalmente el $\langle R\rangle$.

\section{Datos requeridos}

Este modelo se desarrolló con datos extraídos del diseño del reactor a escala laboratorio (Rubio Sierra, Ruiz y Sandoval, 2014). Los principales datos del diseño base se resumen en la tabla 1:

En el diseño de fotobiorreactores airlift, la relación de áreas para el riser y el downcomer, $\mathrm{A}_{\mathrm{r}} / \mathrm{A}_{\mathrm{d}}$, se debería aproximar a 1 (Merchuk, 2003) para evitar pérdidas en el crecimiento algal provocadas por zonas muertas generadas por una mala geometría y una mala agitación, así como por la incidencia insuficiente de luz al sistema. Merchuk (2003) reporta que para diámetros de trabajo inferiores a $0.2 \mathrm{~m}$ se logra un crecimiento algal adecuado respecto a la relación $A_{r} / A_{d}$ con alturas de $1 \mathrm{~m}$. Para la simulación del modelo se toma como constante la relación del diseño base de 0.41.

El incremento de la intensidad de luz al nivel de saturación aumenta la tasa de crecimiento, pero intensidades más altas de luz puede causar fotoinhibición. Longitudes de onda visibles (380$750 \mathrm{~nm}$ ) contribuyen a la fotosíntesis y afectan la tasa de crecimiento de las microalgas (Barsanti y Gualteri, 2014). 
Tabla 1. Especificaciones del diseño base

\begin{tabular}{lc}
\hline \multicolumn{1}{c}{ Parámetro } & Valor \\
\hline Relación $\mathrm{A}_{\mathrm{r}} \mathbf{A}_{\mathrm{d}}$ & 0.41 \\
Velocidad del difusor (CFM) & 64.09 \\
Flujo luminoso (Lux) & 1994 \\
Irradiancia (W/m $\left.{ }^{2}\right)$ & 12.1 \\
Volumen de Operación $\left(\mathrm{cm}^{3}\right)$ & 5600 \\
Volumen Total $(\mathrm{L})$ & 7 \\
Altura de operación (cm) & 54.77 \\
Altura total (cm) & 70 \\
Diámetro de down-comer $(\mathrm{m})$ & 0.114 \\
Diámetro de riser $(\mathrm{m})$ & 0.073 \\
Área del down-comer $\left(\mathrm{m}^{2}\right)$ & $9.5 \times 10^{-3}$ \\
Área del riser $\left(\mathrm{m}^{2}\right)$ & $4.5 \times 10^{-3}$ \\
\hline
\end{tabular}

El espectro de acción fotosintética de Chlorella vulgaris muestra dos preferencias de absorción: azul $(420-450 \mathrm{~nm})$ y rojo $(660-700 \mathrm{~nm})$, con un valor aproximado de intensidad requerida por el alga de 2000 lux $/ \mathrm{m}^{2}$. En concordancia con lo anterior, se trabajó con rangos de energía suministrada entre 60 y $200 \mathrm{~W} / \mathrm{m}^{2}$ en longitudes de onda en el espectro rojo.

El espectro de absorción para diferentes concentraciones de Chlorella vulgaris es estudiado en detalle por Yun y Park (2001). Esta investigación encontró que la Chlorella vulgaris logra una mayor absorción de luz a concentraciones de $1.71 \mathrm{~kg} / \mathrm{m}^{3}$; resultado que fue tomado en cuenta para las concentraciones iniciales suministradas en el modelo.

Para la solución de las ecuaciones del modelo de Cornet se requiere de los parámetros expuestos en la tabla 2, que dependen de la microalga en cuestión.

Tabla 2. Constantes requeridas en el modelo de escalamiento

\begin{tabular}{lcl}
\hline \multicolumn{1}{c}{ Parametro } & Valor & \multicolumn{1}{c}{ Fuente } \\
\hline Punto de compensación $\left(\mathrm{Cp}-\mathrm{W} / \mathrm{m}^{2}\right)$ & 1 & (Cornet et ál,. 1995) \\
$\begin{array}{l}\text { Coeficiente de masa de absorción global }\left(\mathrm{Ea}-\mathrm{m}^{2} / \mathrm{kg}\right) \\
\text { Coeficiente de masa de dispersión global }\left(\mathrm{Es}-\mathrm{m}^{2} / \mathrm{kg}\right)\end{array}$ & 21.4 & (Yun y Park, 2001) \\
$\begin{array}{l}\text { Constante media de saturación para la energía radiante } \\
\text { disponible }\left(\mathrm{k}-\mathrm{W} / \mathrm{m}^{2}\right)\end{array}$ & 4557 & (Yun y Park, 2001) \\
$\begin{array}{l}\text { Velocidad máxima de crecimiento }\left(\mu \mathrm{m}-\mathrm{h}^{-1}\right) \\
\text { Contenido de ficocianinas }\left(\mathrm{Cfc}-\mathrm{kg} / \mathrm{m}^{3}\right)\end{array}$ & 0.049 & (Sasi et ál., 2011) \\
\hline
\end{tabular}

La simulación se llevó a cabo en varias etapas. La primera etapa consistió en hallar el valor de concentración de biomasa $\delta$ y la energía radiante disponible para el cultivo; parámetro que depende de la concentración inicial de biomasa. Este parámetro se calculó para una geometría cilíndrica con base en el modelo de Cornet et ál. (1992), lo que permitió definir rangos de concentración de 
biomasa y la energía radiante disponible para Chorella vulgaris en esos rangos. Las ecuaciones 11 y 12 se resolvieron por el método de Simpson 1/3.

\section{Resultados Y discusión}

\section{Simulación del modelo}

El modelo de Cornet se aplicó para una geometría cilíndrica. Se obtuvo el comportamiento de $4 \pi J_{r} / F_{r}$ vs. $Z$ por medio de las ecuaciones 1,2 y 3 y variando el parámetro $\delta$ para el microorganismo empleado: Chlorella vulgaris. En la figura 1 se muestran los resultados con los rangos de $\delta$ con los que el modelo es reproducible. Como se puede observar, este rango de valores de $\delta$ está entre 7 y 12

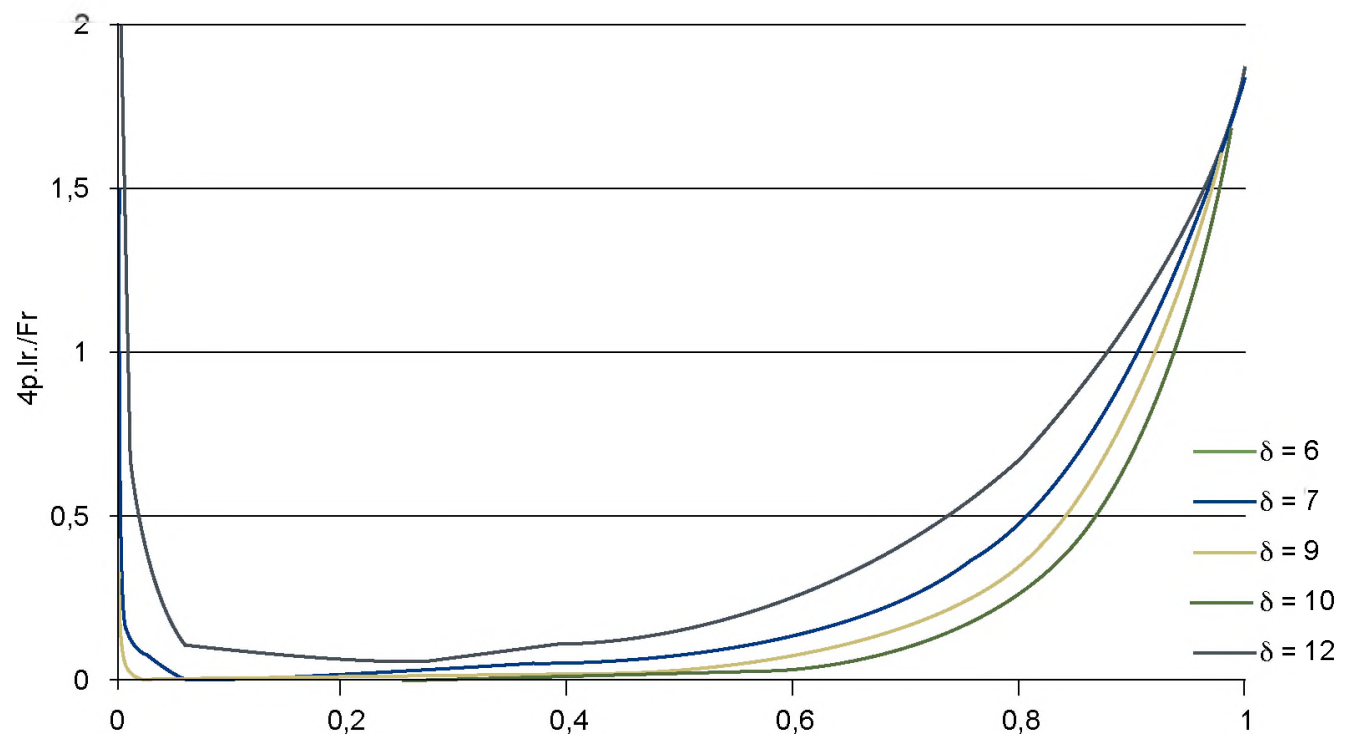

Figura 1. Gráfico de energía disponible $\left(4 \pi J / F_{\mathrm{r}}\right)$ vs. Z para Chlorella vulgaris.

Para valores bajos de $\delta$ (bajas concentraciones de biomasa), la energía disponible desde la pared se concentra en el centro del reactor. Para altas concentraciones de biomasa, los perfiles disminuyen, excepto cerca del centro del reactor $(r=0)$, donde $4 \pi J_{r}$ tiende a ser infinito. Para valores intermedios de $\delta$, los perfiles de energía radiante disponible están en una fase de disminución; luego pasan a una fase cada vez mayor cuando se aproxima al centro del reactor.

A fin de realizar la simulación de modelo, se seleccionaron diámetros (D) del reactor, de 0.114, 0.16 y $0.22 \mathrm{~m}$, con flujos de energía (F) entre los 2 y $300\left(\mathrm{~W} / \mathrm{m}^{2}\right)$. En las tablas 3,4 y 5 se muestran los resultados de la simulación con los respectivos diámetros mencionados. 
Tabla 3. Resultados simulación diámetro $0.114 \mathrm{~m}$ (diámetro del diseño base)

\begin{tabular}{cccccc}
\hline $\begin{array}{c}\mathbf{C x} \\
\left(\mathbf{k g} / \mathbf{m}^{3}\right)\end{array}$ & $\begin{array}{c}\mathbf{F} \\
\left(\mathbf{W} / \mathbf{m}^{2}\right)\end{array}$ & $\begin{array}{c}\mathbf{A} \\
\left(\mathbf{W} / \mathbf{m}^{3}\right)\end{array}$ & $\mathbf{E b}\left(\mathbf{W} / \mathbf{m}^{2}\right)$ & $\begin{array}{c}Y \\
(\%)\end{array}$ & $<\mathbf{R}>\mathbf{( k g / \mathbf { m } ^ { 3 } \mathbf { h } )}$ \\
\hline 0.25 & 2 & 4.49 & 0.447 & 67.1 & 0.000524 \\
0.5 & 12 & 53.9 & 0.0255 & 64.9 & 0.000764 \\
0.5 & 50 & 224.5 & 0.106 & 85.34 & 0.0012 \\
0.5 & 100 & 449.1 & 0.213 & 93.4 & 0.00139 \\
0.7 & 200 & 673.6 & 0.00218 & 75.8 & 0.00113 \\
\hline
\end{tabular}

Tabla 4. Resultados simulación diámetro $0.16 \mathrm{~m}$

\begin{tabular}{cccccc}
\hline Cx $\left(\mathbf{k g} / \mathbf{m}^{3}\right)$ & $\mathrm{F}\left(\mathbf{W} / \mathbf{m}^{2}\right)$ & $\mathbf{A}\left(\mathbf{W} / \mathbf{m}^{3}\right)$ & $E b\left(\mathbf{W} / \mathbf{m}^{2}\right)$ & $\begin{array}{c}\Upsilon \\
(\%)\end{array}$ & $<\mathrm{R}>\left(\mathbf{k g} / \mathbf{m}^{3} \mathbf{h}\right)$ \\
\hline 0.4 & 100 & 320 & 0.135 & 85.5 & 0.00126 \\
0.4 & 200 & 639.9 & 0.271 & 92.2 & 0.00142 \\
0.5 & 200 & 639.9 & 0.0263 & $78 ., 3$ & 0.00119 \\
0.5 & 300 & 959.9 & 0.0245 & 81.8 & 0.00127 \\
\hline
\end{tabular}

Tabla 5. Resultados simulación diámetro $0.22 \mathrm{~m}$

\begin{tabular}{cccccc}
\hline Cx (kg/m3) & $F(\mathbf{W} / \mathbf{m} 2)$ & $\mathbf{A}(\mathbf{W} / \mathbf{m} 3)$ & Eb $(\mathrm{W} / \mathbf{m} 2)$ & $\begin{array}{c}Y \\
(\%)\end{array}$ & $<R>(\mathbf{k g} / \mathbf{m} 3 \mathbf{h})$ \\
\hline 0.27 & 150 & 349.1 & 1.05 & 94.7 & 0.00145 \\
0.27 & 200 & 465.4 & 1.4 & 98.5 & 0.00152 \\
0.3 & 200 & 465.4 & 0.516 & 90.2 & 0.00139 \\
0.3 & 300 & 698.1 & 2.1 & 93.7 & 0.00147 \\
\hline
\end{tabular}

Para los diámetros de 0.16 y $0.22 \mathrm{~m}$ las concentraciones de biomasa $(\mathrm{Cx})$ que validan el modelo de $\delta$ entre 7 y 12 disminuyeron respecto al diseño base, pero siguieron cumpliendo el criterio de la concentración de biomasa descrito anteriormente.

La tabla 3 muestra que para el diámetro del diseño base del reactor, el mayor rendimiento $\left(1,39 \mathrm{E}-03 \mathrm{~kg} / \mathrm{m}^{3} \mathrm{~h}\right)$ se obtuvo con una concentración inicial $(\mathrm{Cx})$ de $0.5 \mathrm{~kg} / \mathrm{m}^{3}$ de biomasa y una energía radiante incidente $(\mathrm{F})$ de $100 \mathrm{~W} / \mathrm{m}^{2}$. Obviamente, a mayor rendimiento, mayor fracción iluminada, $(Y)$, que en ese caso fue del $93 \%$.

Comparando las tablas 4 y 5 , se observa que la energía absorbida (A) presentó una mayor dependencia de la concentración inicial $(\mathrm{Cx})$ que de la energía suministrada $(\mathrm{F})$, ya que se generó la misma cantidad de energía absorbida de $465.4 \mathrm{~W} / \mathrm{m}^{3}$ (para el $\mathrm{D}$ de $0.22 \mathrm{~m}$ ) y $639.9 \mathrm{~W} / \mathrm{m}^{3}$ (para el D de $0.16 \mathrm{~m}$ ) que partió del mismo flujo de energía $\left(200 \mathrm{~W} / \mathrm{m}^{2}\right)$, pero que tuvo diferente concentración inicial: entre 0.4 y $0.5 \mathrm{~kg} / \mathrm{m}^{3}$, para el D de $0.16 \mathrm{~m}$; y 0.27 y $0.3 \mathrm{~kg} / \mathrm{m}^{3}$, para el D de $0.22 \mathrm{~m}$. La diferencia radica en la energía disponible en el centro del reactor $(\mathrm{Eb})$ y la fracción iluminada $(Y)$, que disminuyeron al aumentar la concentración inicial, ya que la demanda lumínica del microorganismo aumentaba con la concentración.

Con el fin de disminuir la cantidad de energía suministrada al reactor $(F)$ para obtener el mayor valor posible de crecimiento volumétrico promedio (R), se seleccionó el diámetro de $0.22 \mathrm{~m}$ que proporcionó valores altos de producción de biomasa, a partir de una baja concentración inicial (Cx) y un relativamente bajo flujo de energía (ver tabla 5).

Con base en este diámetro seleccionado se obtuvieron las dimensiones del reactor escalado que se pueden observar en la tabla 6 , cuyo plano a escala se observa en la figura 2 junto con el reactor inicial. 
Tabla 6. Resultados del volumen escalado

\begin{tabular}{lcc}
\hline \multicolumn{1}{c}{ Descripción } & Unidad & Valor \\
\hline Diámetro del riser & $\mathrm{m}$ & 0.141 \\
Diámetro del down-comer & $\mathrm{m}$ & 0.22 \\
Área del riser & $\mathrm{m}$ & 0.0156 \\
Área del down-comer & $\mathrm{m}$ & 0.038 \\
Altura & $\mathrm{m}$ & 1.32 \\
Altura de operación & $\mathrm{m}$ & 1.06 \\
Volumen total & $\mathrm{L}$ & 50.2 \\
Volumen de operación & $\mathrm{L}$ & 40.1 \\
A/A ${ }_{\mathrm{d}}$ & - & 0.41 \\
Fracción iluminada & - & $98.5 \%$ \\
Crecimiento volumétrico & $\mathrm{kg} / \mathrm{m}^{3} \mathrm{~h}$ & 0.00152 \\
\hline
\end{tabular}

Con respecto al diseño base, la simulación del modelo permitió el escalamiento del reactor desde 7 hasta $50.2 \mathrm{~L}$ de volumen total, con las siguientes mejoras expresadas como incrementos: energía disponible en el centro del reactor, Eb, desde 0.0255 hasta $1.4 \mathrm{~W} / \mathrm{m}^{2}$; porcentaje de fracción volumétrica iluminada desde 64.9 a $98.5 \%$; y crecimiento volumétrico, R, desde $7.64 \times 10^{-4}$ a $1.52 \times 10^{-3} \mathrm{~kg} / \mathrm{m}^{3} \mathrm{~h}$. Estos incrementos se lograron con una concentración inicial de biomasa microalgal de $0.27 \mathrm{~kg} / \mathrm{m}^{3}$, mucho menor a la usada en el diseño base $\left(0.5 \mathrm{~kg} / \mathrm{m}^{3}\right)$.
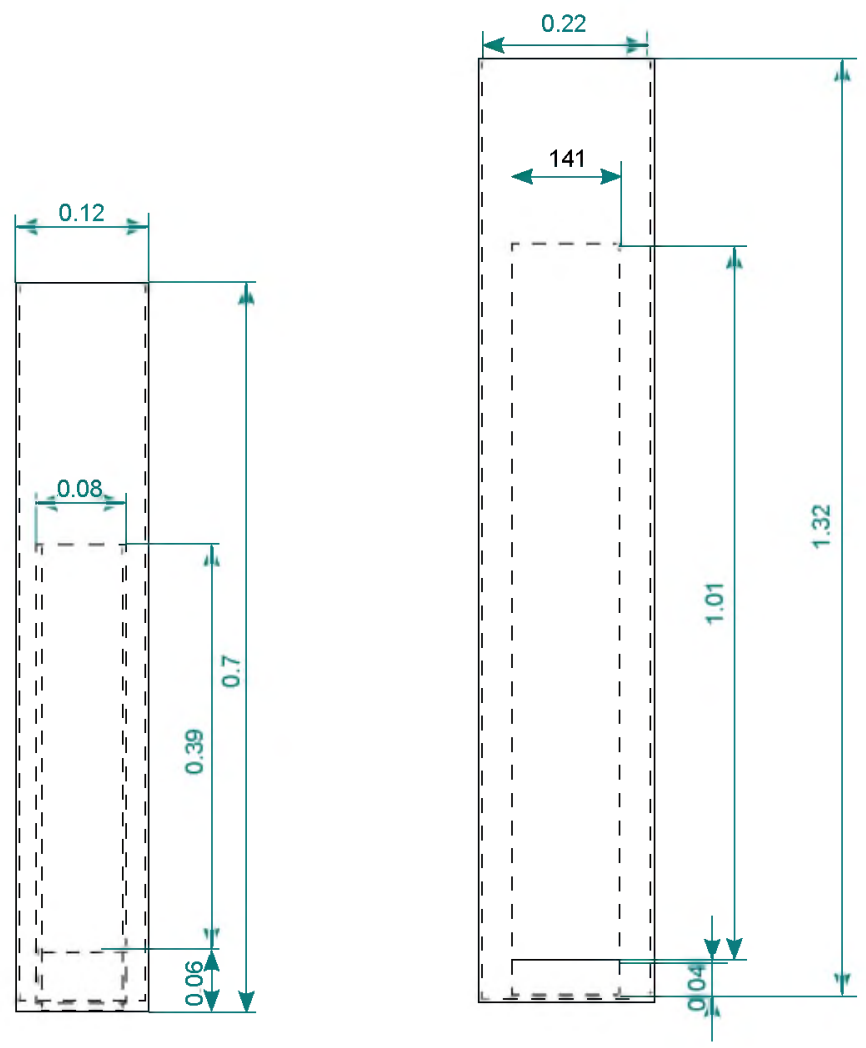

Figura 2. Vistas reactor inicial (a) y final (b). 


\section{Conclusiones}

E1 modelo de escalamiento seleccionado se fundamentó en el fenómeno de la dispersión y absorción de luz acoplado al crecimiento microalgal dentro del fotobiorreactor a través de ecuaciones de balance simplificadas para la determinación de un volumen operacional, lo que constituyó la variable a dimensionar para lograr el escalamiento.

Con un aumento del tamaño del fotobiorreactor de 7 a $50.2 \mathrm{~L}$, que equivale a un aumento del volumen de 7.2 veces aproximadamente, se alcanzó un crecimiento volumétrico de $1.52 \times 10^{-3} \mathrm{~kg} /$ $\mathrm{m}^{3} \mathrm{~h}$ en un sistema de reactor batch, que pese a que no logra superar al crecimiento volumétrico simulado, según referencia bibliográfica, con la cepa Spirulina platensis con valores de $5.95 \times 10^{-3}$ $\mathrm{kg} / \mathrm{m}^{3} \mathrm{~h}$ en un sistema de reactor continuo, sí supera el crecimiento simulado para el fotobiorreactor de escala inferior equivalente a $7.64 \times 10^{-4} \mathrm{~kg} / \mathrm{m}^{3} \mathrm{~h}$, que fue tomado como punto de referencia para el escalamiento. Además, este incremento volumétrico y de rendimiento se logra partiendo de una menor concentración de biomasa microalgal.

\section{REFERENCIAS}

Anaya-Durand, A., y Pedroza-Flores, H. (2008). Escalamiento, el arte de la ingeniería química: plantas piloto, el paso entre el huevo y la gallina. Tecnología, Ciencia, Educación, 23(1), 31-39.

Barrón, V., y Torrent, J. (1986). Use of the Kubelka-Munk theory to study the influence of iron oxides on soil colour. European Journal of Soil Science, 37(4), 499-510.

Barsanti, L., y Gualtieri, P. (2014). Algae: anatomy, biochemistry, and biotechnology. Pisa, Italia: CRC press.

Cornet, J., Dussap, C., y Dubertret, G. (1992). A structured model for simulation of cultures of the cyanobacterium Spirulina platensis in photobioreactors: I. Coupling between light transfer and growth kinetics. Biotechnology and bioengineering, 40(7), 817-825

Hadj-Romdhane, F., Jaouen, P., Pruvost, J., Grizeau, D., Van Vooren, G., y Bourseau, P. (2012). Development and validation of a minimal growth medium for recycling Chlorella vulgaris culture. Bioresource technology, 123, 366-374.

Jeon, Y., Cho, C., y Yun, Y. (2005). Measurement of microalgal photosynthetic activity depending on light intensity and quality. Biochemical Engineering Journal, 27(2), 127-131.

Merchuk, J. (2003). Airlift Bioreactors: a Review of Recent Advances. Canadian journal of chemical engineering, 81(3/4), 324-337.

Najafpour, G. (2015). Biochemical engineering and biotechnology. Bélgica: Elsevier.

Posten, C. (2009). Design principles of photo-bioreactors for cultivation of microalgae. Engineering in Life Sciences, 9(3), 165-177.

Rubio, D., Sierra, J., Ruiz, S., y Sandoval, J. (2014). Diseño de un fotobiorreactor airlift a escala banco. Elementos, 4(4), 123-143.

Sasi, D., Mitra, P., Vigueras, A., y Hill, G. (2011). Growth kinetics and lipid production using Chlorella vulgaris in a circulating loop photobioreactor. Journal of Chemical Technology and Biotechnology, 86(6), 875-880.

Vanags, J., Kunga, L., Dubencovs, K., Galvanauskas, V., y Grīgs, O. (2015). Influence of light intensity and temperature on cultivation of microalgae Desmodesmus Communis in flasks 
and laboratory-scale stirred tank photobioreactor. Latvian Journal of Physics and Technical Sciences, 52(2), 59-70.

van Liere, L., y MUR, L. (1979). Growth kinetics of Oscillatoria agardhii gomont in continuous culture, limited in its growth by the light energy supply. Microbiology, 115(1), 153-160.

Vernerey, A., Albiol, J., Lasseur, C., y Godia, F. (2001). Scale-up and design of a pilot-plant photobioreactor for the continuous culture of Spirulina platensis. Biotechnology progress, 17(3), 431-438.

Yun, Y., y Park, J. (2001). Attenuation of monochromatic and polychromatic lights in Chlorella vulgaris suspensions. Applied microbiology and biotechnology, 55(6), 765-770.

Yun, Y., y Park, J. (2003). Kinetic modeling of the light-dependent photosynthetic activity of the green microalga Chlorella vulgaris. Biotechnology and bioengineering, 83(3), 303-311. 